Document downloaded from:

http://hdl.handle.net/10251/60260

This paper must be cited as:

González Pintor, S.; Ginestar Peiro, D.; Verdú Martín, GJ. (2014). Preconditioning the solution of the time- dependent neutron diffusion equation by recycling Krylov subspaces. International Journal of Computer Mathematics. 91(1):42-52. doi:10.1080/00207160.2013.771181.

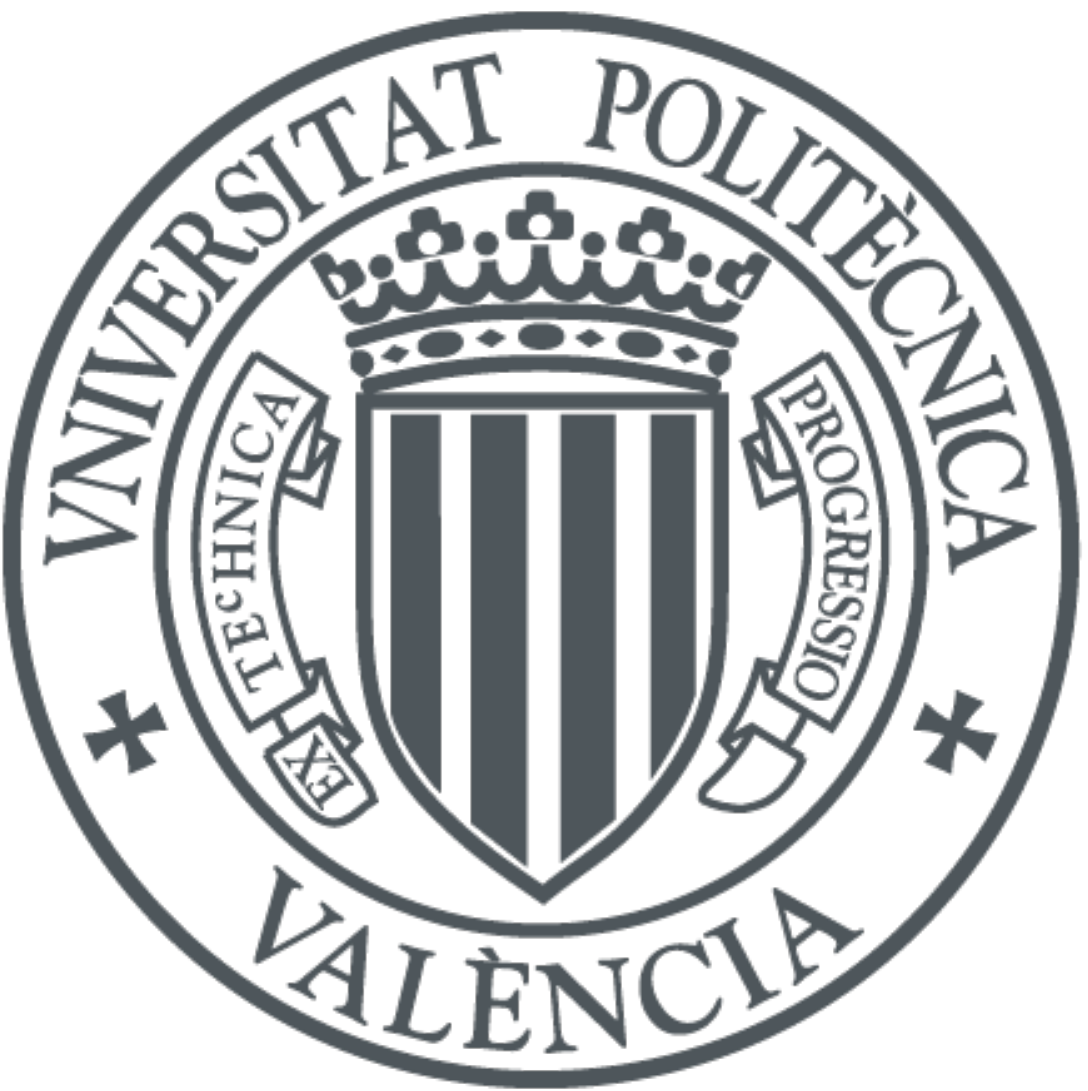

The final publication is available at

http://dx.doi.org/10.1080/00207160.2013.771181

Copyright Taylor \& Francis

Additional Information 


\title{
Preconditioning the solution of the time dependent neutron diffusion equation by recycling Krylov subspaces
}

\author{
S. González-Pintor ${ }^{\mathrm{a}}$, D. Ginestar ${ }^{\mathrm{b}}$ and G. Verdú ${ }^{\mathrm{b}}$ \\ a Instituto de Seguridad Industrial, Radiofísica y Medioambiental; ${ }^{\mathrm{b}}$ Instituto de \\ Matemática Multidisciplinar,
}

Universitat Politècnica de València. Camino de Vera, 14. 46021 València. Spain

(Received 00 Month 200x; in final form 00 Month 200x)

\begin{abstract}
Spectral preconditioners are based on the fact that the convergence rate of Krylov subspace methods is improved if the eigenvalues of smallest magnitude of the system matrix are 'removed'. In this paper, two preconditioning strategies are studied to solve a set of linear systems associated with the numerical integration of the time dependent neutron diffusion equation. Both strategies can be implemented using the matrix-vector product as the main operation and succeed at reducing the total number of iterations needed to solve the set of systems.
\end{abstract}

Keywords: Iterative methods, matrix free preconditioners, Krylov subspace recycling, neutron diffusion equation, time integration.

\section{Introduction}

Nuclear plants are thermal-power systems that generate electricity using the heat generated by nuclear reactions induced by neutrons which take place inside the nuclear reactor. For safe operation and to develop new designs of this kind of plants it is important to have fast and accurate codes that simulate its behaviour. These simulators consist mainly of two different blocks or modules, which solve the models implemented to give account of the basic phenomena taking place in the plant. In this way, there is a neutronic module that simulates the neutron balance in the reactor core and the thermal-hydraulics module, which simulates the heat transfer from the fuel to the water used as coolant and the different condensation and evaporation processes that take place in the core and the condenser systems.

We will focus on the neutronic module. In this way, for a given transient, the neutron balance in the reactor core is modelled using the time dependent neutron diffusion equation in the approximation of two groups of energy [7],

$$
\begin{aligned}
{\left[v^{-1}\right] \frac{\partial \Phi}{\partial t}+\mathcal{L} \Phi } & =(1-\beta) \mathcal{M} \Phi+\sum_{k=1}^{K_{p}} \lambda_{k} \chi \mathcal{C}_{k} \\
\frac{\partial \mathcal{C}_{k}}{\partial t} & =\beta_{k}\left[\begin{array}{lll}
\nu \Sigma_{f 1} & \nu \Sigma_{f 2}
\end{array}\right] \Phi-\lambda_{k} \mathcal{C}_{k}, \quad k=1, \ldots, K_{p}
\end{aligned}
$$

\footnotetext{
*Corresponding author. Email: dginesta@mat.upv.es
} 
where, $K_{p}$ is the number of delayed neutron precursor groups considered,

$$
\mathcal{L}=\left[\begin{array}{cc}
-\vec{\nabla} \cdot\left(D_{1} \vec{\nabla}\right)+\Sigma_{a 1}+\Sigma_{12} & 0 \\
-\Sigma_{12} & -\vec{\nabla} \cdot\left(D_{2} \vec{\nabla}\right)+\Sigma_{a 2}
\end{array}\right], \quad\left[v^{-1}\right]=\left[\begin{array}{cc}
\frac{1}{v_{1}} & 0 \\
0 & \frac{1}{v_{2}}
\end{array}\right]
$$

and

$$
\mathcal{M}=\left[\begin{array}{cc}
\nu \Sigma_{f 1} & \nu \Sigma_{f 2} \\
0 & 0
\end{array}\right], \quad \Phi=\left[\begin{array}{l}
\Phi_{1} \\
\Phi_{2}
\end{array}\right], \quad \chi=\left[\begin{array}{l}
1 \\
0
\end{array}\right]
$$

The diffusion constants and cross-sections, $D_{g}, \Sigma_{12}, \Sigma_{a g}, \nu \Sigma_{f g}, g=1,2$, appearing in the equations depend on the reactor materials, that is, they are position and time dependent functions.

To solve problem (2), a spatial and temporal discretization of the equations has to be selected.

\section{Problem discretization}

\subsection{Spatial discretization}

In a nuclear reactor core, the spatial mesh is naturally defined by the different fuel bundle compositions of the core. Different geometries of the fuel bundles can be be found in different kind of reactors. Thus the fuel elements of western reactors as the PWR or BWR type are rectangular prisms and for example, a nodal collocation method based on a prismatic mesh can be used for its discretization [8]. Reactors such as the russian VVER reactors have fuel bundles with hexagonal geometry. In this case, it is possible to use a high order finite element method [4].

After the spatial discretization process, we obtain a semidiscrete system of ordinary differential equations as follows

$$
\begin{aligned}
{\left[v^{-1}\right] \dot{\psi}+L \psi } & =(1-\beta) M \psi+\sum_{k=1}^{K} \lambda_{k} X C_{k}, \\
X \dot{C}_{k} & =\beta_{k} M \psi-\lambda_{k} X C_{k}
\end{aligned}
$$

where matrices $L, M$ and $X$ are the matrices associated with for the previous differential operators.

\subsection{Time discretization}

The system of ordinary differential equations (4) is a stiff one. Thus, for the time discretization of this system is convenient to use an implicit method. Particularly, a one-step implicit finite differences method is considered [2]. This method consists of solving the above ordinary differential equations (3) and (4) over a series of time intervals $\left[t_{n}, t_{n+1}\right]$. To solve these equations from $t_{n}$ to $t_{n+1}$, first we suppose that the term $M \psi$ varies linearly between these instants, and we approximate the equation (4) as

$$
\begin{aligned}
X \dot{C}_{k}= & -\lambda_{k} X C_{k}+\beta_{k} M^{n} \psi^{n} \\
& +\frac{\beta_{k}}{\Delta t}\left(t-t_{n}\right)\left(M^{n+1} \psi^{n+1}-M^{n} \psi^{n}\right),
\end{aligned}
$$


where $\Delta t=t_{n+1}-t_{n}$, and $M^{n} \psi^{n}$ is $M \psi$ evaluated at time $t_{n}$.

Integrating (5), the solution $X C_{k}$ in $t_{n+1}$ can be expressed as

$$
X C_{k}^{n+1}=X C_{k}^{n} e^{-\lambda_{k} \Delta t}+\beta_{k}\left(a_{k} M^{n} \psi^{n}+b_{k} M^{n+1} \psi^{n+1}\right),
$$

where the coefficients $a_{k}$ and $b_{k}$ are given by

$$
\begin{aligned}
a_{k} & =\frac{\left(1+\lambda_{k} \Delta t\right)\left(1-e^{-\lambda_{k} \Delta t}\right)}{\lambda_{k}^{2} \Delta t}-\frac{1}{\lambda_{k}}, \\
b_{k} & =\frac{\lambda_{k} \Delta t-1+e^{-\lambda_{k} \Delta t}}{\lambda_{k}^{2} \Delta t} .
\end{aligned}
$$

Using backward Euler method in equation (3), we obtain

$$
\begin{aligned}
& {\left[v^{-1}\right] \frac{1}{\Delta t}\left(\psi^{n+1}-\psi^{n}\right)+L^{n+1} \psi^{n+1} } \\
= & (1-\beta) M^{n+1} \psi^{n+1}+\sum_{k=1}^{K} \lambda_{k} X C_{k}^{n+1} .
\end{aligned}
$$

Taking into account equation (6), we reexpress (7) as the system of linear equations

$$
\left[T^{n+1}\right] \psi^{n+1}=\left[R^{n}\right] \psi^{n}+\sum_{k=1}^{K} \lambda_{k} e^{-\lambda_{k} \Delta t} X\left[C_{k}^{n}\right]
$$

where the matrices are defined as

$$
\begin{aligned}
{\left[T^{n+1}\right] } & =\frac{1}{\Delta t}\left[v^{-1}\right]+L^{n+1}-\hat{b} M^{n+1}, \\
{\left[R^{n}\right] } & =\frac{1}{\Delta t}\left[v^{-1}\right]+\hat{a} M^{n},
\end{aligned}
$$

the coefficients $\hat{a}$ and $\hat{b}$ are

$$
\hat{a}=\sum_{k=1}^{K} \lambda_{k} \beta_{k} a_{k}, \quad \hat{b}=1-\beta+\sum_{k=1}^{K} \lambda_{k} \beta_{k} b_{k}
$$

$\Delta t=t_{n+1}-t_{n}$, and $a_{k}$ and $b_{k}$ are given by [2].

$$
a_{k}=\frac{\left(1+\lambda_{k} \Delta t_{n}\right)\left(1-e^{-\lambda_{k} \Delta t_{n}}\right)}{\lambda_{k}^{2} \Delta t_{n}}-\frac{1}{\lambda_{k}}, \quad b_{k}=\frac{\lambda_{k} \Delta t_{n}-1+e^{-\lambda_{k} \Delta t_{n}}}{\lambda_{k}^{2} \Delta t_{n}} .
$$

Then, for each time step, a system of linear equations has to be solved. These systems are large and sparse and to solve then an iterative Krylov method is used, but these methods suffer from slow convergence unless a preconditioner is used. The most popular preconditioners for linear systems are based on incomplete factorizations of the coefficient matrix [6]. Incomplete $L U$ preconditioners are robust, but for very large matrices they are very expensive from the point of view of the 
memory needed for their storage. In this way, alternative methodologies are proposed here that can be implemented using only vector-matrix products and have not storage problems.

\section{Spectral preconditioners}

It is known that the rate of convergence of Krylov subspace methods depend of the distribution of the eigenvalues of the system matrix together with its eigenvectors [3]. In many cases that the convergence of these methods can be greatly improved if the smallest eigenvalues of the matrix are shifted. Spectral preconditioners use this idea to improve the convergence implementing transformations on the linear system, in such a way that the matrix obtained after the spectral transformation has its eigenvalues shifted away from zero. The spectral transformations can be implemented using matrix-vector products and can be applied without the necessity of building explicitly the system matrix [3].

Here, we study methods for preconditioning a sequence of linear systems based on modifying the eigenvalues distribution of the coefficient matrices, by using the information provided by the Krylov subspaces previously computed. Two strategies have been implemented and its performance has been compared to solve the systems associated with the numerical solution of the time dependent neutron diffusion equation. One precondition methodology is based on low rank corrections on the system matrix that modify the eigenvalues distribution of this matrix and another methodology based on recycling an invariant subspace using the the FGCRO-DR method.

To solve the time dependent neutron diffusion equation, it is necessary to solve a sequence of linear systems

$$
A^{n} x^{n}=b^{n}, \quad n=1,2, \ldots
$$

In many transients can be assumed that the spectral properties of the system matrix vary slowly in time, and they are similar from one linear system to the next one. In this way, the invariant subspace associated with the preconditioned matrix at linear system $n$ will be used to precondition the linear system $n+1$.

Invariant subspaces of dimension $k$ should be calculated to precondition the systems. One possibility is to use the GMRES-DR method [5], which in each external iteration recycles an approximate invariant subspace to deflate eigenvalues of smallest magnitude. In particular, to obtain approximate invariant subspaces we have used a variant of GMRES-DR method known as the FGMRES-DR method [1]. The algorithm known as FGMRES $(m)$ to solve a system

$$
A x=b,
$$

of dimension $n$, relies on the Arnoldi relation

$$
\begin{aligned}
& A Z_{m}=V_{m+1} \bar{H}_{m}, \\
& Z_{m}^{H} Z_{m}=I_{m}, \\
& V_{m+1}^{H} V_{m+1}=I_{m+1} .
\end{aligned}
$$

with $Z_{m} \in \mathbb{C}^{n \times m}, V_{m+1} \in \mathbb{C}^{n \times(m+1)}$ has orthonormal columns and $\bar{H}_{m} \in \mathbb{C}^{(m+1) \times m}$ is an upper Hessemberg matrix. Columns of $V_{m+1}$ form an orthonormal basis of 
the subspace spanned by the following vectors

$$
\left\{r_{0}, A z_{1}, \ldots, A z_{m}\right\}
$$

with $r_{0}=b-A x_{0}, Z_{m}=\left[z_{1}, \ldots, z_{m}\right]$ and $V_{m}=\left[v_{1}, \ldots, v_{m}\right]$ are related by

$$
Z_{m}=\left[\mathcal{M}_{j}\left(v_{1}\right), \ldots, \mathcal{M}_{j}\left(v_{m}\right)\right] \quad \text { with } \quad v_{1}=\frac{r_{0}}{\left\|r_{0}\right\|}
$$

where $\mathcal{M}(v)$ is the action of a preconditioner $\mathcal{M}$ on $v$.

To obtain approximations of $k$ eigenvalues of the matrix $A$ and their corresponding eigenvectors, the harmonic Ritz pairs of $A,\left(\lambda_{j}, V_{m} g_{j}\right)$, are obtained solving

$$
\left(H_{m}+\left|h_{m+1, m}\right|^{2} H_{m}^{-H} e_{m} e_{m}^{T}\right) g_{j}=\lambda_{j} g_{j}
$$

which is an eigenvalue problem of small dimension.

\subsection{Low-rank transformations preconditioning}

Let us consider a system

$$
A x=b
$$

and $M$ an initial preconditioner.

Considering $V$ the matrix associated with a right invariant subspace of the matrix $A M$ of dimension $k$

$$
A M V=V J_{k}
$$

where the eigenvalues of $J_{k}$ are $\left\{\lambda_{1}, \ldots, \lambda_{k}\right\}$, the following theorem is satisfied, [3] Theorem 3.1 Let $W$ be such that $A_{c}=W A M V$ is non singular. Defining

$$
M^{(2)}=M+M V A_{c}^{-1} W
$$

the eigenvalues of $A M^{(2)}$ are

$$
\begin{cases}\eta_{i}^{(2)}=\lambda_{i}+1 & \text { if } i \leq k \\ \eta_{i}^{(2)}=\lambda_{i} & \text { if } i>k\end{cases}
$$

In our implementation we have chosen $W^{(j)}=V^{(j) H}$.

This preconditioner allows to shift the lowest (in magnitude) $k$ eigenvalues of the system matrix to be solved and can be applied in a recursive way as follows,

$$
M^{(l+1)}=M+\sum_{j=1}^{l} M^{(j)} V^{(j)}\left(W^{(j)} A M^{(j)} V^{(j)}\right)^{-1} W^{(j)} .
$$

To maintain the required memory by the preconditioner under a user-defined 


\begin{tabular}{llll} 
Table 1. & Types of materials \\
\hline 1 & Fuel & 22 & Mixed \\
2 & Absorber & 23 & Mixed \\
3 & Reflector & 24 & Mixed \\
\hline
\end{tabular}

bound, it will be used in the following form

$$
M^{(l+1)}=M+\sum_{j=1}^{L_{\max }} M^{(j)} V^{(j)}\left(W^{(j)} A M^{(j)} V^{(j)}\right)^{-1} W^{(j)}
$$

where the $L_{\max }$ terms

$$
M^{(j)} V^{(j)}\left(W^{(j)} A M^{(j)} V^{(j)}\right)^{-1} W^{(j)}
$$

are the ones associated with the $L_{\max }$ smallest eigenvalues of the matrix to optimize the performance of the preconditioner.

\section{$3.2 \quad F G C R O-D R$ method}

Flexible GCRO-DR method [1] is an inner-outer method that combines GCRO as the outer method and Flexible GMRES-DR as the inner method, and it allows deflated restarting and subspace recycling.

GCRO method is used to compute optimal approximation over a given set of search vectors in the sense that the residual is minimized, and the inner method FGMRES-DR computes a new search vector by approximately solving the residual equation. GCRO uses two matrices, $U_{k}=\left(u_{1}, \ldots u_{k}\right)$ and $C_{k}=A U_{k}$, with the property that $C_{k}^{T} C_{k}=I_{k}$, and solves the minimization problem

$$
\min _{x \in \operatorname{span}\left(U_{k}\right)}\|b-A x\|
$$

with solution $x=U_{k} C_{k}^{T} b$, and the residual $r=b-A x$ satisfies $r=b-C_{k} C_{k}^{T} b, \quad r \perp$ $\operatorname{span}\left(C_{k}\right)$.

To preserve optimality with respect to the search directions of the outer method (GCRO), the inner method (FGMRES-DR) uses the operator $\left(I-C_{k} C_{k}^{T}\right) A$ instead of $A$, preserving the orthogonality relations from GCRO also in the inner method.

During the process the Ritz pairs of the system matrix can be computed and this spectral information can be easily adapted to a new linear system as the initial search direction of the outer methods thus, the spectral information can be recycled from one system to the next one in a natural way.

\section{Numerical Results}

To test the preconditioning strategies exposed above for the neutron diffusion equation, we have considered a transient in a small reactor of type VVER. The initial configuration for the material distribution in the core is shown in Figures 1(a) and 1(b). There are three different materials, denoted by the numbers 1 , 2 and 3 depending on their type (see Table 1), and other three mixed materials (22, 23 and 24) used to denote the initial axial position of the simple types (see Fig 1(b)) on the radial configuration of the reactor (see Fig 1(a)).

The transient defined is defined as follows: 


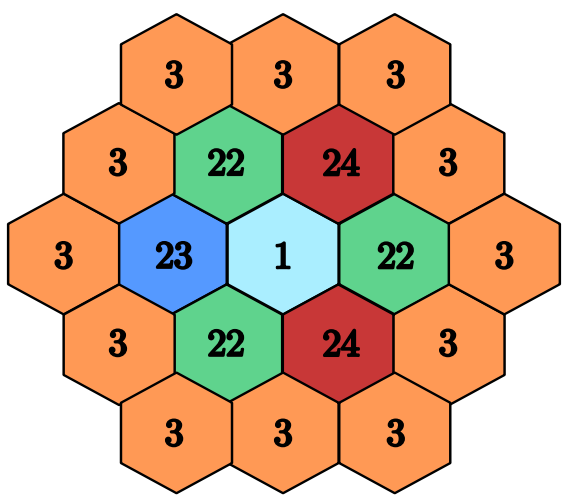

(a) Axial plane

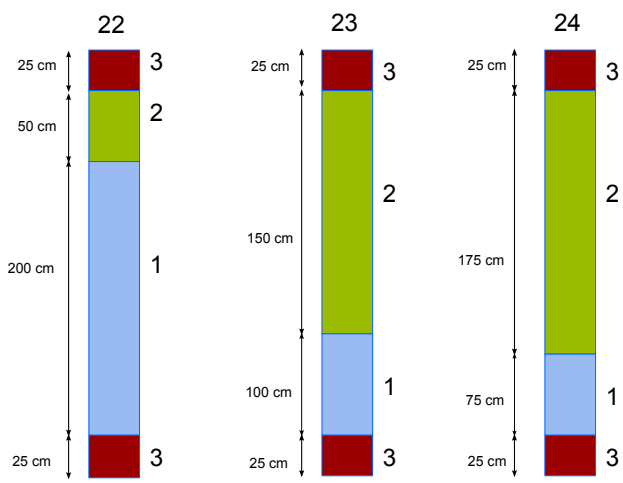

(b) Axial nodes at initial configuration

Figure 1. Small reactor

- At time $t=0.0 \mathrm{~s}$, starting form the initial configuration, the height of the absorber (material 2) at position 23 becomes smaller at constant velocity until it is completely removed at time $t=0.15 s$, remaining only the fuel (material 1 ) in the unrodded cells, simulating a rod ejection accident.

- From time $t=0.15 \mathrm{~s}$ until time $t=1.0 \mathrm{~s}$ nothing happens.

- When the security system acts, a scram is produced inserting absorbers at positions 22 from time $t=1.0 \mathrm{~s}$ until the bottom of the reactor is reached at time $t=9.0 s$.

Evolution of the normalized mean power, starting from a critical situation of the reactor, is monitored until time $t=7.0 \mathrm{~s}$. Figure 4 shows the evolution of the power during the transient.

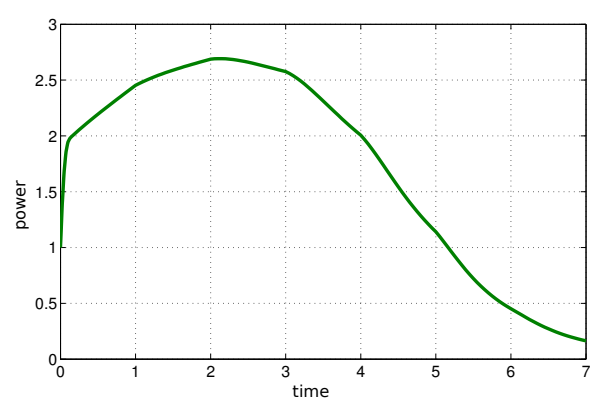

Figure 2. Power along the transient.

From this transient we have considered 80 systems of linear equations corresponding to the time interval $[0,1] \mathrm{s}$ with a time step $\Delta t=0.0125 \mathrm{~s}$.

First, for the spatial discretization with the high order finite element method, we have considered $K_{x, y}=1$ and $k_{z}=1$ in the polynomial expansions obtaining matrices of dimension $N=1898$ with $\mathrm{NNZ}=65860$ non-zero elements (linear discretization), and $K_{x, y}=2$ and $k_{z}=2$ in the polynomial expansions obtaining matrices of dimension $N=12950$ with $\mathrm{NNZ}=1064284$ non-zero elements (quadratic discretization).

The the low rank transformations preconditioning is used to update an initial preconditioner $M$, which is taken initially as the diagonal of the matrix. This preconditioner is used in combination with $\operatorname{FGMRES-DR}(m, k)$ method. The number of iterations needed to solve each of the systems values of $L_{\max }=15$ and $L_{\max }=25$ 


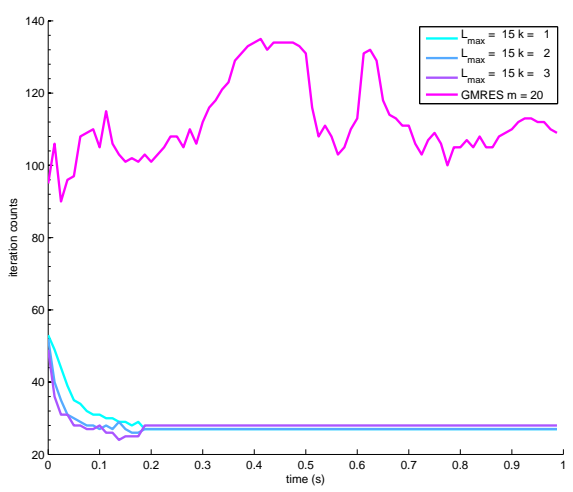

(a) Linear discretization

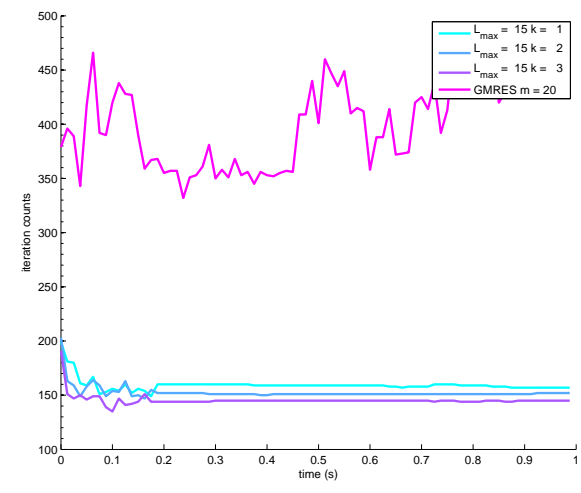

(b) Quadratic discretization

Figure 3. Number of iterations used by the preconditioned $\operatorname{FGMRES-DR}(m, k)$ with different values for the dimension $k$ of the recycled space, setting $L_{\max }=15$.

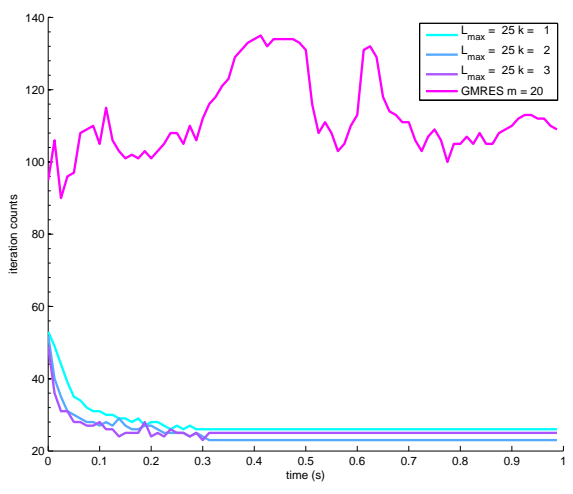

(a) Linear discretization

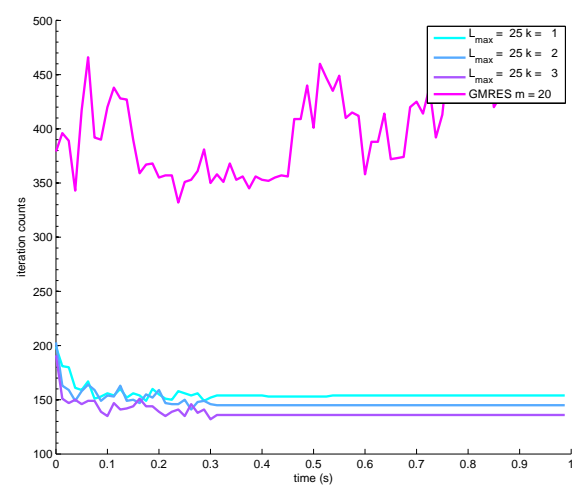

(b) Quadratic discretization

Figure 4. Number of iterations used by the preconditioned FGMRES-DR $(m, k)$ with different values for the dimension $k$ of the recycled space, setting $L_{\max }=25$.

using different values of $k$ are shown in Figure 3 and in Figure 4, for linear and quadratic spatial discretizations. These results have been obtained with a fixed dimension $m=20$ of the restarting Krylov subspace. For comparison, the results of solving the same transient with the classical GMRES $(m)$ method, that is the same that taking $L_{\max }=0$ and $k=0$ with the FGMRES-DR $(m, k)$, is included. There can be observed the improvement of using FGMRES-DR $(m, k)$ with the low rak transformations preconditioning, even when recycling little information. It can be also observed than the improvement scales well with the size of the matrix systems.

The same set of systems has been solved using FGCRO-DR $(m, k)$ method and an approximate invariant subspace associated with the smallest eigenvalues of one system matrix is recycled to precondition the solution of the next system. The number of iterations needed to solve each system setting the dimension of the restarted Krylov subspace to $m=20$ and $m=40$ for different values of the dimension of the recycled space, $k$, are shown in Figures 5 and 6 , respectively, for linear and quadratic spatial discretizations.

In all the cases the use of the spectral preconditioners is efficient to reduce the total number of iterations needed to solve the total set of linear systems associated with the transient in the reactor. 


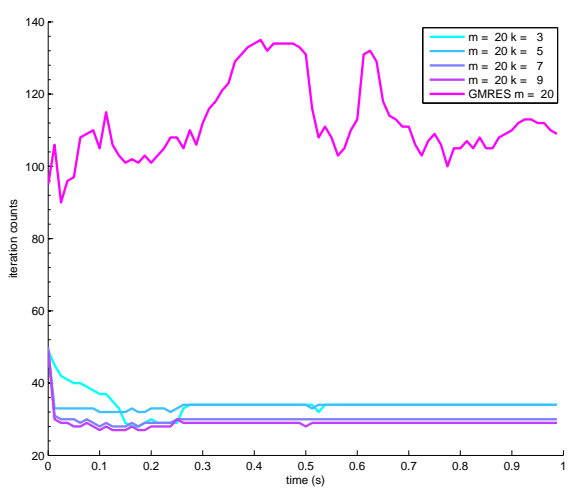

(a) Linear discretization

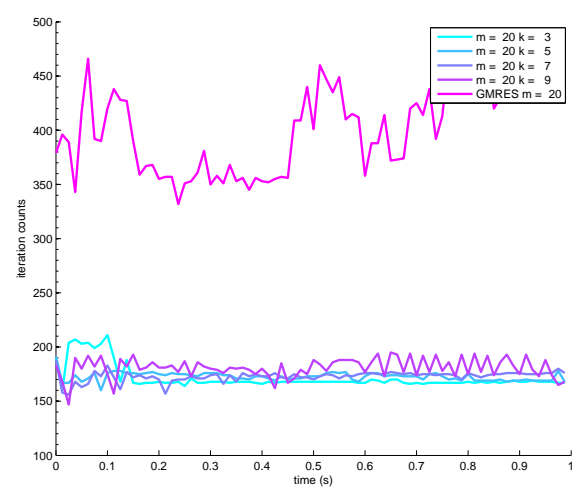

(b) Quadratic discretization

Figure 5. Number of iterations needed by FGCRO-DR $(m, k)$ method setting $m=20$ with different combinations for the dimension $k$ of the recycled space.

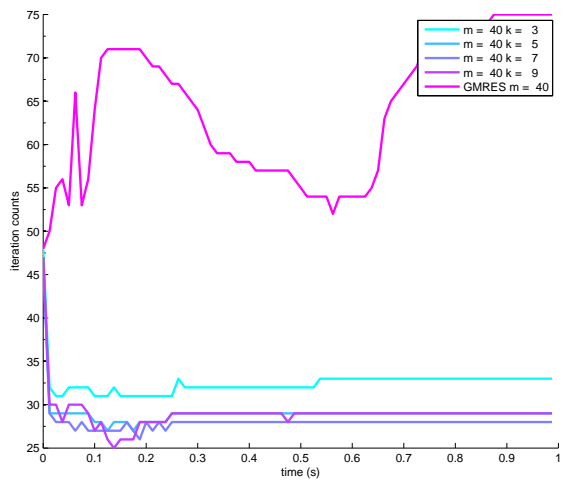

(a) Linear discretization

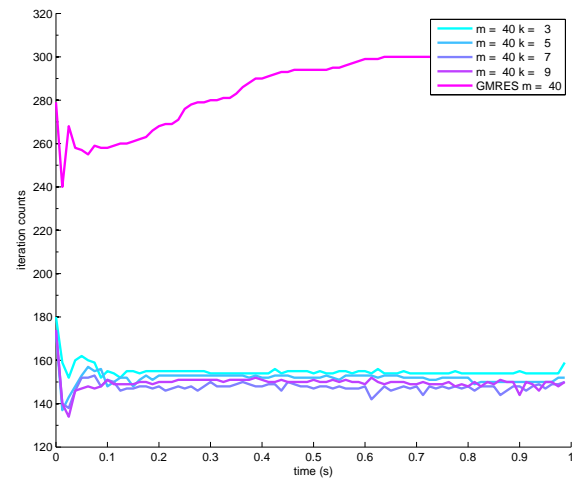

(b) Quadratic discretization

Figure 6. Number of iterations needed by FGCRO-DR $(m, k)$ method setting $m=40$ with different combinations for the dimension $k$ of the recycled space.

\section{Conclusions}

In this paper two spectral methodologies to precondition the solution of a set of linear systems are analyzed using sets of linear systems arising in the simulation of a transient in a nuclear power reactor using the time dependent neutron diffusion equation. The first methodology uses low rank transformations to update an initial preconditioner to shift the smallest eigenvalues of the system matrix. This preconditioning technique is combined with the FGMRES-DR $(m, k)$ method. The second methodology uses the recycling of an approximate invariant subspace associated with the smallest eigenvalues of a system matrix to precondition the solution of the next system. This second methodology is implemented using the FGCRO$\operatorname{DR}(m, k)$. Both methodologies have been tested with two sets of linear systems associated with the numerical solution of the time dependent neutron diffusion equation. In the studied cases it is observed that the use of these preconditioners succeeds reducing the total number of iterations needed to solve the set of linear systems associated with the transient. 


\section{Acknowledgments}

This work has been partially supported by the Spanish Ministerio de Educación y Ciencia under projects MTM2010-18674 and ENE2011-22823.

\section{References}

[1] L. M. Carvalho, S. Gratton, R. Lago, X. Vasseur, A flexible generalized conjugate residual method with inner orthogonalization and deflated restarting. SIAM J. Matrix Anal. Appl. 32, 4, (2011), pp. $1212-1235$.

[2] D. Ginestar, G. Verdú, V. Vidal, R. Bru, J. Marín, J.L. Muñoz-Cobo, High Order Backward Discretization of the Neutron Diffusion Equation, Annals of Nuclear Energy 25 (1-3) (1998), pp. 47-64.

[3] L. Giraud, S. Gratton, E. Martin, Incremental spectral preconditioners for sequences of linear systems, Applied Numerical Mathematics 57 (2007), pp. 1164-1180.

[4] S. González-Pintor, D. Ginestar, G. Verdú, High Order Finite Element Method for the Lambda Modes problem on hexagonal geometry. Annals of Nuclear Energy 36 (2009), pp. 1450-1462.

[5] R. B. Morgan, GMRES with defated restarting, SIAM J. Sci. Comput., 24 (2002), pp. 20-37.

[6] Y. Saad. Iterative Methods for Sparse Linear Systems, SIAM, Philadelphia, 2003.

[7] W.M. Stacey, Nuclear Reactor Physics, John Wiley \& Sons Inc, New York, 2001.

[8] G. Verdú, D. Ginestar, V. Vidal, J.L. Muñoz-Cobo, 3D- $\lambda$ modes of the neutron diffusion equation, Annals of Nuclear Energy 21,7 (1994), pp. 405-421. 